\title{
A measurement-based Fault Detection Approach Applied To Monitor Robots Swarm
}

\author{
${ }^{(1)}$ Belkacem Khaldi, ${ }^{(2)}$ Fouzi Harrou, Member, IEEE, ${ }^{(2)}$ Ying Sun, ${ }^{(1)}$ Foudil Cherif
}

\begin{abstract}
Swarm robotics requires continuous monitoring to detect abnormal events and to sustain normal operations. Indeed, swarm robotics with one or more faulty robots leads to degradation of performances complying with the target requirements. This paper present an innovative data-driven fault detection method for monitoring robots swarm. The method combines the flexibility of principal component analysis (PCA) models and the greater sensitivity of the exponentiallyweighted moving average control chart to incipient changes. We illustrate through simulated data collected from the ARGoS simulator that a significant improvement in fault detection can be obtained by using the proposed methods as compared to the use of the conventional PCA-based methods.
\end{abstract}

\section{INTRODUCTION}

\section{A. The state of the art}

In recent decades, swarm intelligence techniques in multirobotics systems are becoming among the fastest growing areas in the field of robotics [1], [2]. Swarm robotics, which has been recently attracted attention of scientists and engineers in various disciplines, has been involved in mimicking intelligent swarming of social animals like foraging, aggregation, flocking, cooperative objects transportation, and self-organized patterns formation [3]-[5]. Moreover, swarm robotics is very useful for several applications, such as collective detection of bombs, cooperative search and exploration, managing warehouses and delivering products to customers, seeding, harvesting, and storing grains, rescuing human beings in emergency situations.

Practically, accomplishing the assigned tasks with the desired performances using a robots swarm system is not always expected to be properly executed. This fact is mainly due to external interferences or failures resulted either from component faults such as bugs in the robots software controller, electromechanical faults in the robots sensors and actuation devices, topological faults like broken communication links and intrusions between robots of the swarm. Of course, swarm robotics with one or more faulty robots leads to degradation of performances complying with the target requirements. Therefore, it is crucial to be able to detect and identify any possible faults or failures in the monitored swarm robotics system as early as possible. As a result of accurate and prompt fault detection, efficiency and operating

Belkacem Khaldi and Foudil Cherif are with (1) LESIA Laboratory, Department of Computer Science, University of Mohamed Khider, B.P. 145, R.P. 07000 Biskra, Algeria, khaldi.belkacem@gmail.com

Fouzi Harrou and Ying Sun are with ${ }^{(2)}$ King Abdullah University of Science and Technology, Computer, Electrical and Mathematical Sciences and Engineering Division, Thuwal, 23955-6900, Saudi Arabia, fouzi.harrou@kaust.edu.sa capacity of the swarm system are improved, and expense maintenance are avoided.

Generally, fault detection techniques can be categorized into two major classes [6], [7]: endogenous and exogenous fault detection techniques. Endogenous approaches are used to monitor each robot individually to reveal any faults. However, such approaches ignore the interaction between robots and therefore result in a misleading analysis. In the other hand, exogenous fault detection techniques were developed to inspect several robots simultaneously [8]. In other words, in such techniques a robot could detect errors that occurred in other robot components by taking into consideration the available information of its neighborhood in the swarm [9][11]. Both endogenous and exogenous techniques can be developed using mathematical model or empirical implicit model for fault detection. In model-based approaches, faults are detected based on a comparison between the actual behaviors of the monitored system with predicted behaviors derived from a mathematical model of a system [7], [12]. In the absence of an explicit model data-driven models are a suitable alternative [6], [13].

\section{B. Motivation and contributions}

While several fault detection have been reported in the last decades for swarm robotics systems, until recent time multivariate statistical process control charts such as PCA, however, have not been used to monitor swarm robotics. This paper focus on monitoring swarm robots based on PCA-based fault detection approaches. PCA is one the most commonly used technique for dimensionality reduction. Using PCA method, the covariance structure in the data can be explained in a reduced dimensional space through an orthogonal set of principal components, i.e, a set of linear combinations of the original variables. By extracting useful data from the original dataset using PCA modeling and then using monitoring indices faults in the monitored swarm robotics can be detected. Unfortunately, conventional PCAbased monitoring indices such as $T^{2}$ and $Q$ charts are less efficient in detecting incipient changes in the mean of process data [14]-[16].

The first objective is to tackle multivariate challenges in process monitoring by merging the advantages of univariate and traditional multivariate techniques to enhance their performance and widen their practical applicability. EWMA (exponentially-weighted moving average) control chart is widely used univariate control charts. The key idea is to apply PCA dimension reduction techniques to the features of a process, and use control charts to monitor more informative 
variables in a lower dimension. Specifically, we extend the abilities of the univariate monitoring techniques such as EWMA to deal with multivariate processes by developing linear PCA-based EWMA monitoring method to monitor swarm robotics systems. Note that the main advantage of PCA-based EWMA fault detection approach is that the testing step is performed online which is not the case of a classifier (the classifier algorithms are performed offline rather than online). The decision can be provided at each new sample by comparing the value of EWMA decision statistic with the value of the threshold. An anomaly is declared if EWMA statistic exceeds the threshold.

The proposed monitoring approach is applied to detect faults in a swarm of foot-bot robots while they are forming together a regular circle. We refer to the Virtual Viscoelastic Control (VVC) model proposed in [17] for swarm robots circle formation, the model was implemented in a simulated version of e-puck robots using the ARGoS simulator [18]. Here we re-implement the model within the same simulator in a simulated version of foot-bot robots. During simulation running, we collect various inputs/outputs data of each single robots of the swarm, these data are later used in the PCA model for monitoring.

The following section briefly reviews the virtual viscoelastic control Model used for swarm robots circle formation. Section III review the PCA-based approach and how it can merged with EWMA chart and used for fault detection. In Section V, the performances of the proposed methods are illustrated in a simulation study, and Section VI concludes with a discussion and suggestions for future research directions..

\section{ViRTUAL Viscoelastic CONTROL MODEL}

The VVC model is a physics-based model that has been successfully applied as a proximal control to keep and arrange robots together within a certain distance [5], [17]. In this model the movement of the swarm is governed by virtual viscoelastic forces, which result from the interactions of the robots with each other. This allows certain connectivity and coherency between the robots of the swarm while they are on movement. The motion of each robot (its right speed $v_{r i}$ and left speed $v_{l i}$ ) depends on the virtual viscoelastic force $F_{i}^{v v c}$, which is given by the following equation:

$$
F_{i}^{v v c}=\sum_{j=1}^{n}\left(k_{s}\left(d_{i, j}-d_{0}\right)+\mu v_{i, j}\right)
$$

where $d_{0}=2 r \sin (\pi /(n+1)), n$ is the number of neighbors and $r$ is the radius of the desired circle. $k_{s}$ is the spring constant, $d_{i, j}$ is the displacement vector that represents the current length of the spring between two interacting robots, $d_{0}$ is the equilibrium length of the spring, $\mu$ is the damping coefficient, $v_{i, j}$ is the velocity of the focal robot with regards to its nearby mate.

The speeds of the robot' wheels are computed as follows:

$$
\left[\begin{array}{c}
v_{l_{i}} \\
v_{r_{i}}
\end{array}\right]=\left[\begin{array}{cc}
1 & \frac{b}{2} \\
1 & \frac{-b}{2}
\end{array}\right]\left[\begin{array}{c}
v_{i} \\
\omega_{i}
\end{array}\right]
$$

here $b$ is the distance between the robot' wheels. The robot' angular velocity $\omega_{i}$ and the robot' forward speed $v_{i}$ are given as follows:

$$
\omega_{i}=k_{\omega} \angle F_{i}^{v v c}, v_{i}=\frac{v_{\max }}{\sqrt{\left|\omega_{i}\right|+1}}
$$

where $k_{\omega}$ is a gain constant, $\angle F_{i}^{v v c}$ refers to the angle formed by the force $F_{i}^{v v c}$, and $v_{\max }$ is the maximum allowed forward speed.

To achieve the VVC model in a foot-bot robot, we use its range and bearing sensing and communication device (called RAB). With this device, the foot-bot is able to send and receive messages to (from) nearby robots within a maximum range $D_{r}$. Moreover; it can also perceive the range $\left(d_{i j}\right)$ and bearing $\left(\theta_{i j}\right)$ measurements of the robot that sent a message. The model is implemented using the ARGoS simulator, further details about the values of constants and parameters we used in the model can be found in [5], [17].

\section{PCA-BASED MONITORING APPROACHES}

\section{A. Feature Extraction Using PCA}

Consider a properly scaled data matrix or measurement matrix $\mathbf{X}=\left[\mathbf{x}_{1}^{T}, \ldots, \mathbf{x}_{n}^{T}\right]^{T} \in R^{n \times m}$ with $n$ measurements and $m$ process variables. In the following discussion, it is assumed that the scaled data is zero mean centered with unit variance. Usually, due to redundancy and noise in the data, $l$, principal components $(l \ll m)$ can capture much of the variability in $\mathbf{X}$. The data matrix $\mathbf{X}$ can be expressed by PCA as two complementary orthogonal parts: a modeled data $\widehat{\mathbf{X}}$ which contains the most significant variations present in the data and a residual data $\mathbf{E}$ which represents noises, i.e.,

$$
\mathbf{X}_{s}=\mathbf{T P}^{T}=\sum_{i=1}^{l} t_{i} p_{i}^{T}+\sum_{i=l+1}^{m} t_{i} p_{i}^{T}=\widehat{\mathbf{X}}+\mathbf{E}
$$

with $\mathbf{T}=\left[\begin{array}{ll}t_{1} & t_{2} \cdots t_{m}\end{array}\right] \in R^{n \times m}$ represents a matrix of the transformed uncorrelated variables, $t_{i} \in R^{n}$ termed principal components. $l$ is the number of PCs retained in the PCA model. The column vectors $p_{i} \in R^{m}$, termed the loading vectors, arranged in the matrix $\mathbf{P} \in R^{m \times m}$ are obtained by the eigenvectors related to the covariance matrix of $\mathbf{X}_{s}$, i.e., $\Sigma$. The loading vectors are the eigenvectors of the covariance matrix, $\Sigma$. Through singular value decomposition, the covariance matrix, $\Sigma$, can be decomposed as:

$$
\Sigma=\frac{1}{n-1} \mathbf{X}_{s}^{T} \mathbf{X}_{s}=P \Lambda P^{T} \text { with } P P^{T}=P^{T} P=I_{n} .
$$

Here, $\Lambda=\operatorname{diag}\left(\sigma_{1}^{2}, \ldots, \sigma_{m}^{2}\right)$ is a diagonal matrix containing the eigenvalues of $\Sigma$ in decreasing magnitude, and $I_{n}$ is the identity matrix [19]. In PCA, it is very important to select the optimal number of PCs to be retained in the model [20]. Here $l$ is chosen such that there is no significant process information left in $\mathbf{E}$, and $\mathbf{E}$ is expected to contain only the random error. There are many techniques for selecting the dimension $l$, such as cross-validation, cumulative percent variance and variance of reconstruction error. In this paper, 
$\mathrm{CPV}$ technique is used employed to determine the number of retained PCs, $l$,

$$
C P V(l)=\frac{\sum_{i=1}^{l} \lambda_{i}}{\sum_{i=1}^{m} \lambda_{i}} \times 100 .
$$

Once a PCA model based on data representing historical normal operation is obtained, it can be utilized to monitor future deviation of the process from normality. Two monitoring indices, $T^{2}$ and $Q$, are usually used with PCA for fault detection [21]. However, the PCA-based $T^{2}$ and $Q$ approaches fails in detecting small faults. EWMA chart, which is widely used univariate control charts, is proposed as improved alternative for fault detection. In other words, the objective is to tackle PCA challenges in process monitoring by merging the advantages of EWMA and PCA approach to enhance their performance and widen their practical applicability.

\section{UNIVARIATE STATISTICAL CONTROL CHARTS}

The EWMA charts are able to detect small shifts in the process mean as they are time-weighted, since the EWMA statistic is a time-weighted average of all previous observations. The EWMA control scheme was first introduced by Roberts [22], and has been extensively used in time series analysis [23]-[27]. Assume that $\left\{x_{1}, x_{2}, \ldots, x_{n}\right\}$ are individual observation collected from a monitored process. The expression for the EWMA is [27]:

$$
z_{t}=\lambda x_{t}+(1-\lambda) z_{t-1} \quad \text { if } t>0 .
$$

The starting value $z_{0}$ is usually set to be the mean of faultfree data, $\mu_{0} . Z_{t}$ is the output of EWMA and $x_{t}$ is the observation from the monitored process at current time. The forgetting parameter $\lambda \in(0,1]$ determines how fast EWMA forgets the data history. We can see that if $\lambda$ is small, more weight is assigned to past observations and the chart is tuned to have efficiency for detecting small changes in the process mean. On the other hand, if $\lambda$ is chosen to be large, more weight is assigned to the current observations and less weights are assigned to its previous observations, and the chart is suitable to detect large shifts [26], [27]. In the special case, when $\lambda=1$, in which case the EWMA is equal to the most recent observation, $x_{t}$, and provides the same results as Shewhart chart. Under fault free conditions, the standard deviation of $z_{t}$ is defined as $\sigma_{z_{t}}=\sigma_{0} \sqrt{\frac{\lambda}{(2-\lambda)}\left[1-(1-\lambda)^{2 t}\right]}$, where $\sigma_{0}$ is the standard deviation of the fault-free or preliminary data set.The upper and lower control limits of the EWMA chart for detecting a mean shift are: $U C L / L C L=\mu_{0} \pm L \sigma_{z_{t}}$, where $L$ is a multiplier of the EWMA standard deviation $\sigma_{z_{t}}$. The parameters $L$ and $\lambda$ need to be set carefully [26], [27].

\section{A. Combining PCA with EWMA - PCA-EWMA}

Once a PCA model based on data representing historical normal operation is obtained, it can be utilized to monitor future deviation of the process from normality. In this paper, we exploit the advantages of PCA modeling with the univariate monitoring chart EWMA, and this should result in an improved fault detection system, especially for detecting small faults in highly correlated multivariate data. Towards this end, we applied EWMA chart to minor components obtained from PCA model. Minor components refer to the components that are not retained in a PCA model. As we know, the principal components explain must of variation in the data. Only unimportant or residual information is left in minor components. Minor components usually contain residual for the constraints or redundancies that exist between variables. Few studies have taken the minor components into account when doing PCA analysis. The loading vectors related to the minor components actually describe the correlations between variables. The minor components, which capture the variability that arises from noise, represent the residuals of the process. Indeed, under normal operation the minor components are close to zero due to measurement noise and errors, while they significantly deviate from zero under the presence of abnormal events. In this work, the minor components are used as fault indicator. The PCAEWMA fault detection algorithm schematically summarized in Figure 1.

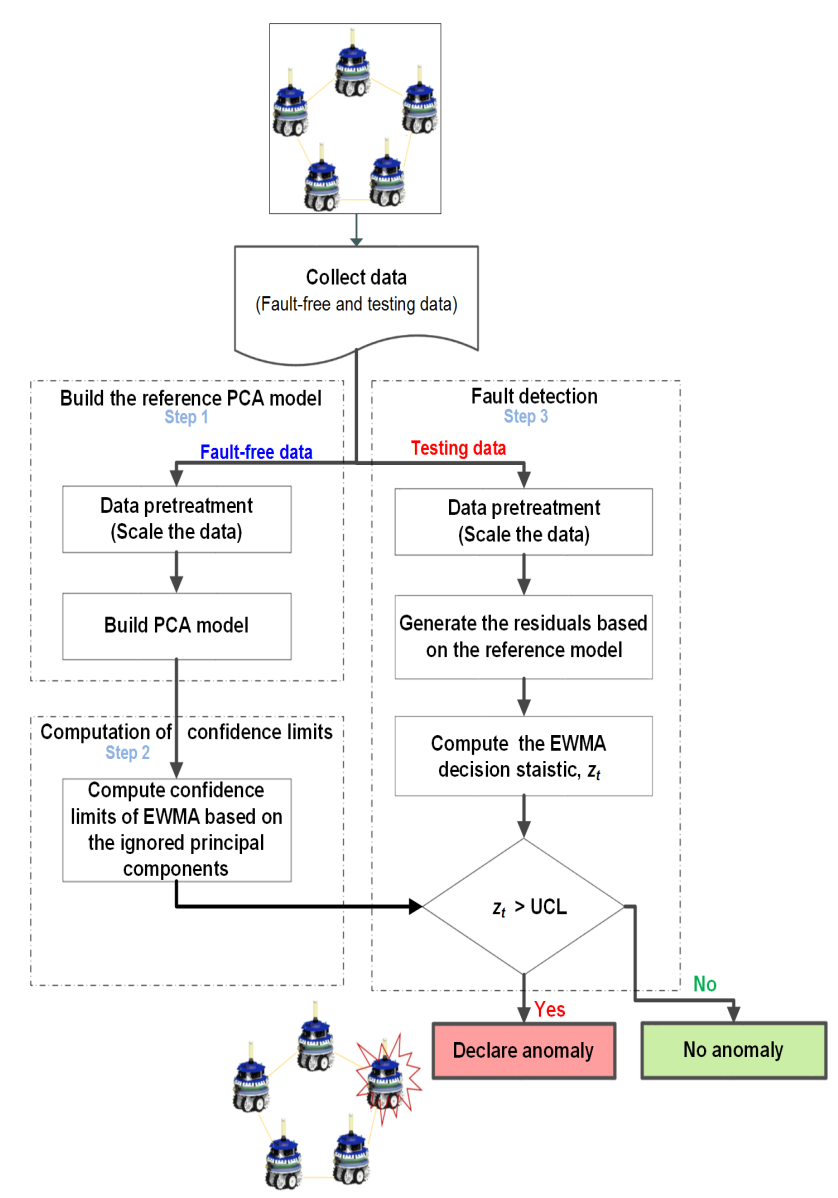

Fig. 1. A flowchart of a PCA-based fault detection scheme. 


\section{Simulation RESUlts}

In this study, we perform ARGoS-based experimental simulations within a swarm of foot-bots; the robots are programmed to perform the VVC model to self-organize themselves into forming a uniform circle when starting from an initial distributions. ARGoS comes with a configuration file in which we can set the arena, the robots and their sensors and actuators devices. In our simulation setup, we activate the RAB equipment within a range $D_{r}=3 m$, we set the arena to be a closed room of $10 * 6 \mathrm{~m}^{2}$, the number of the foot-bots implicated in the simulation is set to $n=6$, the foot-bots are randomly distributed in the arena and their orientations are set to be a Gaussian distribution of zero means and a standard deviation of $360^{\circ}$. In ARGoS, The simulation time step is set to $0.1 s$ long, we perform five runs for the same experiment for a total of 1500 time steps. During this experimental simulations, we collect data about both the virtual viscoelastic force length $F_{i}^{v v c}$ and the virtual viscoelastic force angle $\angle F_{i}^{v v c}$ of each robot of the swarm. These are further used as inputs/outpus for the PCA-based monitoring approach.

\section{A. PCA modeling}

The data matrix $\mathbf{X}$ used to build PCA contains 3000 observations and 12 variables (i.e., viscoelastic force length and viscoelastic force angle collected from each robot). These twelve signals are considered to be measured when the swarm system is operating at normal conditions. First, these training data have been scaled to zero mean and variance one, and then used to build a PCA model. The number of the PCs retained in the PCA model are determined using CPV method with the threshold of $95 \%$. The first PC explains $56 \%$ of the total variance; the second PC explains $37 \%$ of the total variance and the third PC explains $3 \%$ of the total variance. Totally, three PCs can capture almost $96 \%$ of the useful information in the monitored swarm robots system. Thus, only three PCs need to be retained in the PCA model.

Monitoring results of PCA-based $T^{2}, Q$ and EWMA charts for the normal operating data are shown in Figure 2(a)(c). Since the Q plot shown in Figure 2(b) is based on normal operating data, one should expect that almost all the data will lie within $95 \%$ confidence interval. Similarly, the data points in the PCA-EWMA chart are also within the 95 confidence limits (see Figure 2(c)-(d)). However, the $T^{2}$ plot given in Figure 2(a) shows a few false alarms. We can conclude that the PCA model describe well the data where no faults are present.

In this paper, three types of fault will be considered: gradual, intermittent and abrupt faults.

\section{B. Detection results}

1) Abrupt fault detection: In this section, an abrupt change is simulated by adding a small constant deviation to viscoelastic force length of the first robot, $\mathbf{x}_{1}$ between sample times 150-200. Since the viscoelastic force is largely related to the RAB device, this could represent a miss-perception in the range of neighbours or noisy data (velocities) received
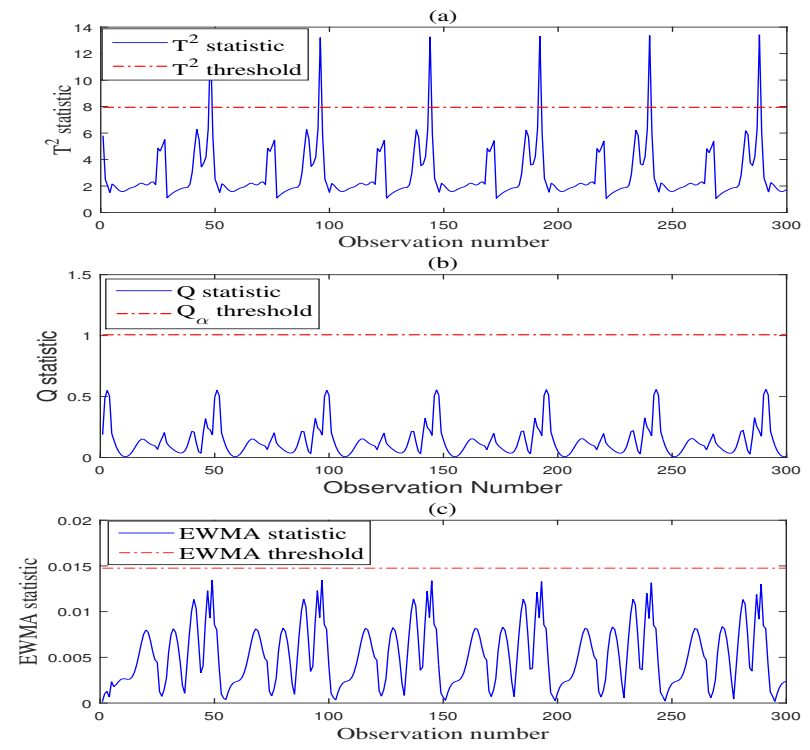

Fig. 2. Monitoring results of PCA-T $T^{2}$ (a), PCA-Q (b) and PCA-EWMA charts(c) for the normal operation data.

from neighbours. The magnitude of the deviation is equal to $40 \%$ of the total variation in $\mathrm{x}_{1}$. Monitoring results are shown in Figure 3(a)-(d). The $T^{2}$, as expected, has no ability to whatsoever to detect this moderate fault (see Figure 3(a)). This fact is due to the PCs space sometimes being insensitive to moderate and small faults, which is because each principal component is a combination of all process variables. The monitoring results of PCA- $Q$, PCAEWMA, charts are demonstrated in Figure 3(b)-(c). From the figure, all charts can give signals of fault because the bias shift in this case is quite large.
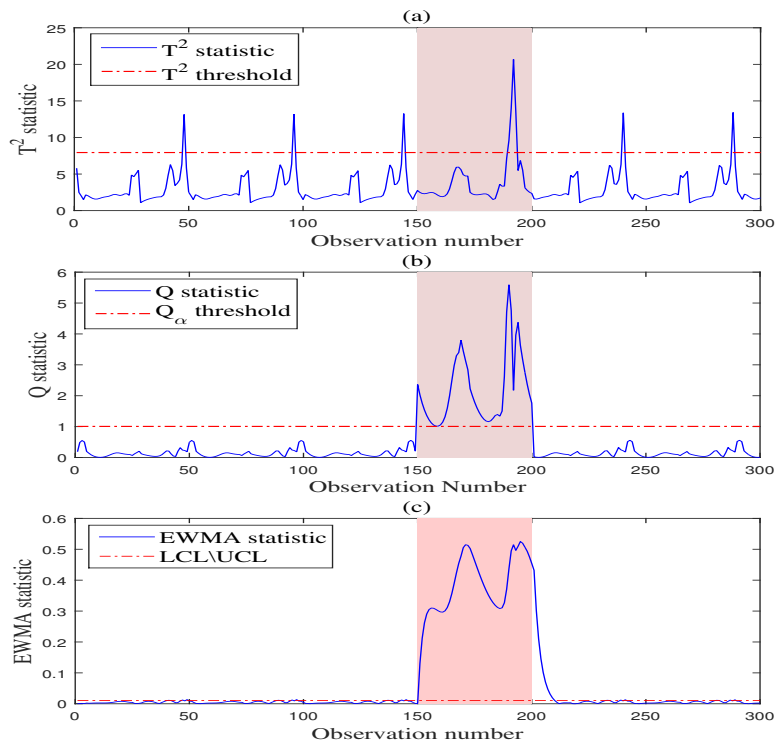

Fig. 3. Monitoring results of the $T^{2}$ (a), $Q$ (b), EWMA with $\lambda=0.3$ (c) charts in the presence of abrupt fault in $x_{1}$ from sample 100 to 150 (Case (i), example 1). 
2) Intermittent fault: In this case study, we introduce into testing data a bias of amplitude $40 \%$ of the total variation in $\mathrm{x}_{1}$ of between samples 50 and 100 and a bias of $10 \%$ from sample 150 to 200 . This again could due to a repeated miss-perception of the range and the bearing measurements to nearby robots or a noisy received data (a RAB sensor fault). Figure 4(a)- (d) shows the monitoring results of the PCA-based $T^{2}, Q$, EWMA charts. Figure 4(a) shows that the PCA-based $T^{2}$ chart has no power to detect this fault. From Figure 4(b), it can be seen that PCA-EWMA chart can detect the intermittent faults but with several missed detections. On the other hand, PCA-EWMA chart with $\lambda=0.3$ can correctly detect this intermittent fault (see Figure 3(c)). In this case study, we can see that detection performance is much enhanced when using PCA-EWMA chart compared to the other charts
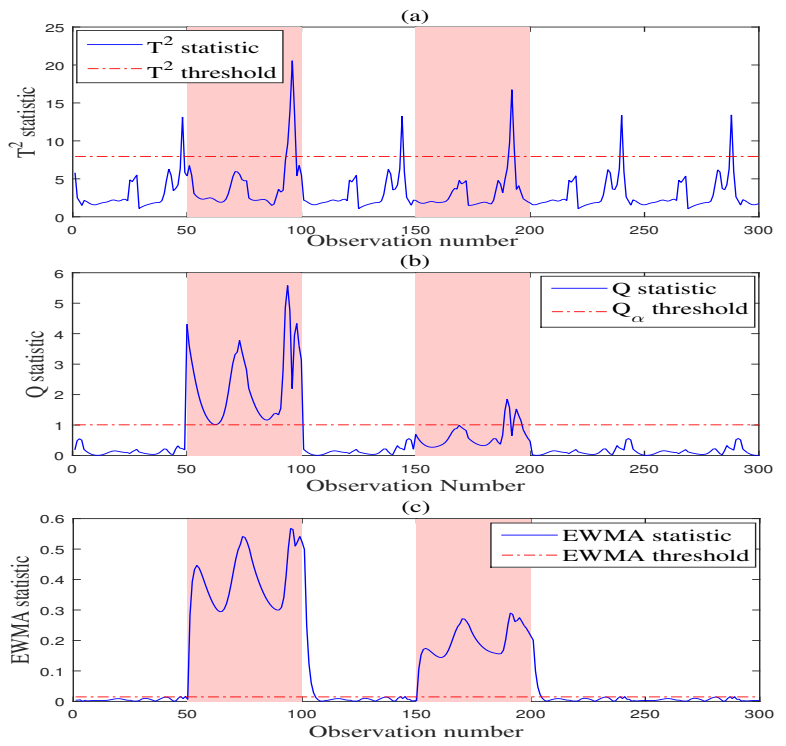

Fig. 4. Monitoring results of the $T^{2}$ (a), $Q$ (b), EWMA with $\lambda=0.3$ (c) charts in the presence of intermittent faults in $x_{1}$ from sample 100 to 150 (Case (i), example 2).

3) Drift failure detection: A ramp type or a slow drift fault is simulated by adding a ramp change to normal measurements of $x_{1}$ from sample 250 till the end of testing data. Figure 5(a) shows that the PCA- $T^{2}$ is not sensitive to this drift fault. The PCA- $Q$ chart is shown in Figure 5(b), which indicates the first signal at the sample 181. Figure 5(c) shows that the PCA-EWMA chart detects the first signal at the 157th observation. Therefore, fewer extra observations are needed for the PCA-EWMA chart to detect a signal compared to the other charts.

\section{CONCLUSION}

The paper focuses on improved data-based fault detection strategy and its application in detecting faults in a swarm of foot-bot robots. Towards this end, the VVC model is used for swarm robots circle formation. Different kind of faults have been tested in this sudy including, abrupt fault and drift fault. It is shown through simulated swarm robots
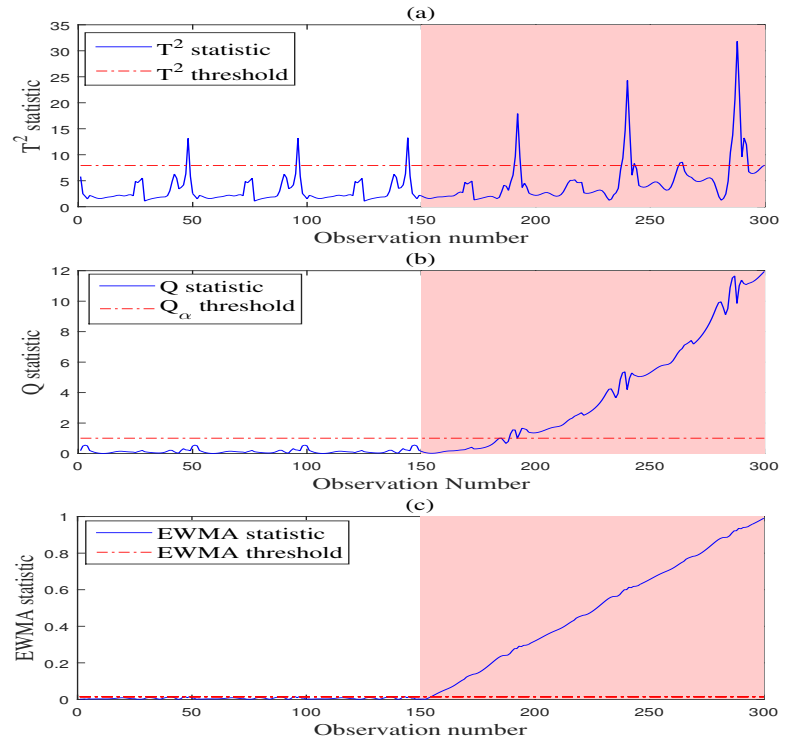

Fig. 5. Monitoring results of the $T^{2}$ (a), $Q$ (b), EWMA with $\lambda=0.3$ (c) charts in the presence of drift fault with slope 0.01 in temperature sensor $x_{1}$ from sample 250

data via the ARGoS simulator that significant improvement in fault detection can be obtained by using the EWMA chart as compared to the use of $Q / T^{2}$ which are conventionally used with PCA based technique. Because the PCA-based $T^{2}$ and $Q$ charts evaluate the monitored system performance based on the observed current data alone, they are suitable at detecting relatively large faults. They are less efficient in detecting relatively small and persistent shifts, compared to EWMA chart.

\section{ACKNOWLEDGEMENT}

This publication is based upon work supported by the King Abdullah University of Science and Technology (KAUST) Office of Sponsored Research (OSR) under Award No: OSR-2015-CRG4-2582. The authors (Belkacem Khaldi and Foudil Cherif) would like to thank the LESIA Laboratory, Department of Computer Science, University of Mohamed Khider,Biskra, Algeria for the continued support during the research.

\section{REFERENCES}

[1] B. Khaldi and F. Cherif, "An overview of swarm robotics: Swarm intelligence applied to multi-robotics," International Journal of Computer Applications, vol. 126, no. 2, 2015.

[2] Y. Tan and Z. Zheng, "Research advance in swarm robotics," Defence Technology, vol. 9, no. 1, pp. 18-39, 2013.

[3] M. Rubenstein, A. Cabrera, J. Werfel, G. Habibi, J. McLurkin, and R. Nagpal, "Collective transport of complex objects by simple robots: theory and experiments," in Proceedings of the 2013 international conference on Autonomous agents and multi-agent systems. International Foundation for Autonomous Agents and Multiagent Systems, 2013, pp. 47-54.

[4] E. Ferrante, A. Turgut, C. Huepe, A. Stranieri, C. Pinciroli, and M. Dorigo, "Self-organized flocking with a mobile robot swarm: a novel motion control method," Adaptive Behavior, p. $1059712312462248,2012$.

[5] B. Khaldi and F. Cherif, "Swarm robots circle formation via a virtual viscoelastic control model," in $8 t h$ International Conference on Modelling, Identification and Control (ICMIC-2016). IEEE, 2016. 
[6] H. Lau, "Error detection in swarm robotics: A focus on adaptivity to dynamic environments," 2012.

[7] E. Skoundrianos and S. Tzafestas, "Finding fault-fault diagnosis on the wheels of a mobile robot using local model neural networks," IEEE Robotics \& Automation Magazine, vol. 11, no. 3, pp. 83-90, 2004.

[8] H. Lau, I. Bate, P. Cairns, and J. Timmis, "Adaptive data-driven error detection in swarm robotics with statistical classifiers," Robotics and Autonomous Systems, vol. 59, no. 12, pp. 1021-1035, 2011.

[9] A. L. Christensen, R. OâĂŹGrady, M. Birattari, and M. Dorigo, "Exogenous fault detection in a collective robotic task," in European Conference on Artificial Life. Springer, 2007, pp. 555-564.

[10] A. Christensen, R. OGrady, and M. Dorigo, "From fireflies to faulttolerant swarms of robots," IEEE Transactions on Evolutionary Computation, vol. 13, no. 4, pp. 754-766, 2009.

[11] A. Khadidos, R. Crowder, and P. Chappell, "Exogenous fault detection and recovery for swarm robotics," IFAC-PapersOnLine, vol. 48, no. 3, pp. 2405-2410, 2015.

[12] R. Canham, A. Jackson, and A. Tyrrell, "Robot error detection using an artificial immune system," in NASA/DoD Conference on Evolvable Hardware. IEEE, 2003, pp. 199-207.

[13] M. Mokhtar, R. Bi, J. Timmis, and A. Tyrrell, "A modified dendritic cell algorithm for on-line error detection in robotic systems," in IEEE Congress on Evolutionary Computation. IEEE, 2009, pp. 2055-2062.

[14] F. Harrou, M. Madakyaru, Y. Sun, and S. Khadraoui, "Improved detection of incipient anomalies via multivariate memory monitoring charts: Application to an air flow heating system," Applied Thermal Engineering, DOI: 10.1016/j.applthermaleng.2016.08.047, 2016.

[15] F. Harrou, F. Kadri, S. Khadraoui, and Y. Sun, "Ozone measurements monitoring using data-based approach," Process Safety and Environmental Protection, vol. 100, pp. 220-231, 2016.

[16] F. Harrou, Y. Sun, and S. Khadraoui, "Amalgamation of anomalydetection indices for enhanced process monitoring," Journal of Loss Prevention in the Process Industries, vol. 40, pp. 365-377, 2016.

[17] B. Khaldi and F. Cherif, "A virtual viscoelastic based aggregation model for self-organization of swarm robots system," in Conference Towards Autonomous Robotic Systems. Springer, 2016, pp. 202-213.

[18] C. Pinciroli, V. Trianni, R. OŠGrady, G. Pini, A. Brutschy, M. Brambilla, N. Mathews, E. Ferrante, G. D. Caro, and F. Ducatelle, "ARGoS: a modular, parallel, multi-engine simulator for multi-robot systems," Swarm intelligence, vol. 6, no. 4, pp. 271-295, 2012.

[19] J. Jackson and G. Mudholkar, "Control procedures for residuals associated with principal component analysis," Technometrics, vol. 21, pp. 341-349, 1979.

[20] M. Zhu and A. Ghodsi, "Automatic dimensionality selection from the scree plot via the use of profile likelihood," Computational Statistics \& Data Analysis, vol. 51, pp. 918-930, 2006.

[21] S. Qin, "Statistical process monitoring: Basics and beyond," Journal of Chemometrics, vol. 17, no. 8/9, pp. 480-502, 2003.

[22] J. Lucas and M. Saccucci, "Exponentially weighted moving average control schemes: properties and enhancements," Technometrics, vol. 32, no. 1 , pp. 1-12, 1990.

[23] F. Harrou and M. Nounou, "Monitoring linear antenna arrays using an exponentially weighted moving average-based fault detection scheme," Systems Science \& Control Engineering: An Open Access Journal, vol. 2, no. 1, pp. 433-443, 2014.

[24] A. Morton, M. Whitby, M. McLaws, A. Dobson, S. McElwain, D. Looke, J. Stackelroth, and A. Sartor, "The application of statistical process control charts to the detection and monitoring of hospitalacquired infections," Journal of quality in clinical practice, vol. 21, no. 4, pp. 112-117, 2001.

[25] F. Harrou, M. Nounou, and H.N.Nounou, "A statistical fault detection strategy using PCA based EWMA control schemes," in 9th Asian Control Conference (ASCC). IEEE, 2013, pp. 1-4.

[26] F. Kadri, F. Harrou, S. Chaabane, Y. Sun, and C. Tahon, "Seasonal arma-based spc charts for anomaly detection: Application to emergency department systems," Neurocomputing, vol. 173, pp. 2102 2114, 2016.

[27] D. C. Montgomery, "Introduction to statistical quality control," John Wiley\& Sons, New York, 2005. 